\title{
FOMENTO DE LAS HABILIDADES SOCIOEMOCIONALES EN EL CURRÍCULO ESCOLAR DE LA EDUCACIÓN SUPERIOR COMO CONTRIBUCIÓN PARA UNA MEJOR CONVIVENCIA
}

\section{PROMOTION OF SOCIO-EMOTIONAL SKILLS IN THE SCHOOL CURRICULUM OF HIGHER EDUCATION AS A CONTRIBUTION TO A BETTER COEXISTENCE}

\author{
Dra. Karina Trejo Sánchez ${ }^{1}$ \\ Universidad Autónoma Metropolitana, Unidad Cuajimalpa
}

\section{RESUMEN}

En México, la educación se encuentra regulada principalmente en el artículo $3^{\circ}$ de la Constitución Política de los Estados Unidos Mexicanos y en la Ley General de Educación. Ambos dispositivos fundamentan la contribución de la educación para la mejor convivencia

\footnotetext{
$1 \quad$ karinats@hotmail.com 5585814853 Universidad Autónoma Metropolitana, Unidad Cuajimalpa. Ciudad de México. Enlace de registro en ORCID: https://orcid.org/0000-0001-6264-5638

Enlace de registro en Google académico:

https://scholar.google.es/citations?view op=list works\&hl=es\&user=s 8CgfMAAAAJ

Posdoctorada por la Universidad Autónoma Metropolitana (UAM), Unidad Xochimilco; Doctora en Derecho con vertiente en Pedagogía, aprobada con mención honorífica por la División de Estudios de Posgrado de la Facultad de Derecho de la UNAM; Maestra de Derecho con vertiente en Pedagogía, por la División de Estudios de Posgrado de la Facultad de Derecho de la UNAM; Licenciada Derecho, por la Facultad de Derecho de la UNAM. Licenciatura en Psicología en Área Educativa, aprobada con mención honorífica por la Facultad de Psicología de la UNAM. Es Profesora Investigadora Titular de Tiempo Completo por tiempo indeterminado, adscrita al Departamento de Estudios Institucionales de la UAM; Coordinadora de la Licenciatura en Derecho de la UAM-Cuajimalpa; Profesora de Posgrado en la Facultad de Humanidades y Ciencias Sociales de la Universidad La Salle, Ciudad de México. Autora del libros y capítulos de libros en editoriales nacionales e internacionales; articulista en revistas arbitradas e indexadas. Coordinadora y conferencista en eventos académicos nacionales e internacionales.
} 
humana. El espacio universitario es un nicho potencial de construcción de vínculos sociales y afectivos, como parte de las necesidades básicas que intenta cubrir del ser humano como ente social. Estos vínculos podrían potenciarse para una mejor convivencia, si desde el currículo escolar de la educación superior se fortaleciera el desarrollo de las habilidades socioemocionales de sus alumnos. El presente ensayo tiene por objetivo evidenciar que el desarrollo de habilidades socioemocionales en alumnos de educación superior puede contribuir a una mejor convivencia, así como plantear una propuesta con un esbozo de las rutas a seguir para fomentar las referidas habilidades en el currículum de la educación superior.

PALABRAS CLAVE: convivencia, currículum escolar, educación superior, habilidades socioemocionales.

\section{ABSTRACT}

In Mexico, education is regulated mainly in Article 3 of the Political Constitution of the United Mexican States and in the General Education Law. Both devices support the contribution of education for better human coexistence. The university space is a potential for the construction of social and affective bonds, as part of the basic needs that the human being tries to cover as a social entity. These links can be improved for a better coexistence, and from the school curriculum of higher education the development of the socio-emotional skills of its students is strengthened. The objective of this essay is to demonstrate the development of socio-emotional skills in higher education students, and to help them to better coexist, as well as to present a proposal with an outline of the routes to be followed in order to promote the aforementioned skills in the higher education curriculum.

KEYWORDS: coexistence, school curriculum, higher education, socio-emotional skills.

\section{PROMOÇÃO DE HABILIDADES SOCIOEMOCIONAIS NO CURRÍCULO ESCOLAR DO ENSINO SUPERIOR COMO CONTRIBUIÇÃO PARA UMA MELHOR CONVIVÊNCIA}

No México, a educação é regulada principalmente no artigo 3 da Constituição Política dos Estados Unidos Mexicanos e na Lei Geral de Educação. Ambos os dispositivos apóiam a contribuição da educação para a melhor coexistência humana. O espaço universitário é um nicho potencial para a construção de laços sociais e emocionais, como parte das necessidades básicas que o ser humano tenta suprir como entidade social. Esses vínculos poderiam ser aprimorados para uma melhor coexistência, se, a partir do currículo escolar do ensino superior, o desenvolvimento das habilidades socioemocionais de seus alunos fosse fortalecido. O objetivo deste ensaio é mostrar que o desenvolvimento de habilidades socioemocionais em estudantes do ensino superior pode contribuir para uma meIhor convivência, bem como propor uma proposta com um esboço das rotas a seguir para promover as habilidades mencionadas no currículo do ensino superior.

PALAVRAS-CHAVE: convivência, currículo escolar, ensino superior, habilidades socioemocionais.

Sumario: I. Introducción. II. Marco conceptual. III. Desarrollo de habilidades socioemocionales en alumnos de educación superior como contribución para una mejor convivencia. IV. Vías para fomentar las habilidades socioemocionales en el currículo escolar de la educación superior. V. Discusión. VI. Conclusiones. VII. Referencias.

\section{INTRODUCCIÓN}

El ser humano es un ser social que sólo resuelve sus necesidades básicas en la relación con los demás. Entre sus necesidades está la de 
construir vínculos afectivos y sociales (MECD, 1998: 26). Estos vínculos podrían potenciarse, si en la educación formal se fortaleciera el desarrollo de las habilidades socioemocionales, lo cual podría contribuir a una mejor convivencia. Ante lo cual, no está exenta la participación de la educación superior.

La idea de propiciar las habilidades socioemocionales en el currículo de la educación superior se fundamenta en la afirmación del Banco Interamericano de Desarrollo (2017) respecto a que las personas no dejan $-\mathrm{y}$ no deberían dejar- de aprender. Y que la acumulación de las habilidades durante la edad adulta forma parte esencial del desarrollo de habilidades en la vida de una persona (p. 265).

En México, la educación se encuentra regulada en el artículo $3^{\circ}$ de la Constitución Política de los Estados Unidos Mexicanos (CPEUM) y en la Ley General de Educación (LGE). Ambos dispositivos fundamentan la contribución de la educación para la mejor convivencia humana.

De ahí que en este texto se pretende responder a las cuestiones: ¿el desarrollo de habilidades socioemocionales en alumnos de educación superior puede contribuir a una mejor convivencia? Y ¿cómo fomentar las habilidades socioemocionales en el currículum de la educación superior? Teniendo como objetivo evidenciar que el desarrollo de habilidades socioemocionales en alumnos de educación superior puede contribuir a una mejor convivencia, así como plantear una propuesta con un esbozo de las rutas a seguir para fomentar las referidas habilidades en el currículum de la educación superior.

Para lograr su cometido, el texto se aborda con el desarrollo de los siguientes ejes: Marco conceptual, desarrollo de habilidades socioemocionales en alumnos de educación superior como contribución para una mejor con- vivencia, vías para fomentar las habilidades socioemocionales en el currículum escolar de la educación superior, discusión y conclusiones.

\section{MARCO CONCEPTUAL}

\section{Habilidades sociales y emocionales}

Para describir qué son las habilidades sociales y emocionales es importante determinar primero que se entiende por habilidad. Al respecto, Justicia y Cano (1996) advierten: "es una disposición para hacer actividades concretas, bien sean de tipo físico, bien sean de tipo mental. Son rutinas que existen para llevar a cabo tareas especificadas y se desarrollan mediante técnicas a través del entrenamiento" (Como se citó en Trianes Torres, M. V., y Gallardo Cruz, J. A., 2008: 444). En ese sentido, se puede deducir que una habilidad se puede adquirir con la práctica y dar como resultado de ésta una destreza en algo determinado.

Por tanto, se dice que alguien es hábil o diestro cuando su comportamiento se ajusta o amolda a las características del objeto o acontecimiento con el que se relaciona (Ribes Iñesta, E., 2002: 197). Esto indica que se genera un dominio a ese respecto. Monereo, C., (1998): afirma que las habilidades son capacidades desarrolladas mediante el uso de procedimientos y pueden utilizarse o ponerse en juego, tanto consciente como inconscientemente, de forma automática (p. 18). De ahí que cuando se adquiere una habilidad ésta es aplicable de manera inmediata.

En virtud de lo anterior, "las habilidades son formas efectivas de actuar que se pueden desarrollar y mejorar" (De la Mora Ledesma, J. G., 1990: 15), lo cual implica que las habilidades se pueden aprender. 
Así, las características del aprendizaje de habilidades son:

- $\quad$ El aprendizaje de una habilidad empieza con un intento de mejorar.

- $\quad$ Necesidades de un modelo.

- $\quad$ El aprendizaje de una habilidad requiere práctica.

- $\quad$ El conocimiento de los resultados (De la Mora Ledesma, J. G., 1990: 16 y 17).

De la Mora Ledesma (1990) sostiene que "el resultado que se espera lograr con este aprendizaje es doble: desarrollar la habilidad y crear hábitos para emplearla en situaciones en que sea apropiada" (p. 15).

Por su parte, "las habilidades sociales $y$ emocionales son el conjunto de habilidades, tanto cognitivas como sociales, que configuran unos comportamientos interpersonales afectivos en los diferentes ámbitos de relación" (Adam, E. et. al., 2007, p. 64).

Para la OCDE (2016) las habilidades sociales y emocionales, se manifiestan en patrones consistentes de pensamientos, sentimientos y comportamientos; se pueden desarrollar mediante experiencias formales e informales de aprendizaje; e influyen en resultados socioeconómicos importantes en la vida de la persona. $Y$ a su vez, contribuyen a lograr objetivos (perseverancia, auto control y pasión por los objetivos), y a trabajar con otros (mediante la sociabilidad, el espeto y la solicitud) así como a manejar de emociones (autoestima, optimismo y confianza) (p. 36).

Respecto a las habilidades sociales, son las capacidades o destrezas sociales específicas requeridas para ejecutar competentemente una tarea interpersonal (García Rojas, A. Daniel, 2010 , p. 226). Zavala Berbena (2008) añade que son conductas observables, aprendidas y utilizadas en los intercambios sociales para obtener fines concretos (p. 326).

En tanto, las habilidades emocionales son destrezas para autorregular las emociones, las cuales "son impulsos que comportan reacciones automáticas y constituyen un conjunto innato de sistemas de adaptación al medio" (Segura Morales, M. y Arcas Cuenca, M., 2007, p. 15).

Los primeros investigadores en establecer una definición sobre las habilidades emocionales fueron John Mayer y Peter Salovey, quienes puntualizaron que se trataba de la destreza para supervisar los sentimientos propios y ajenos, con el fin de lograr discriminarlos y utilizar esta información en la orientación del pensamiento y el comportamiento propio (López de Bernal, M.E. y González Medina, M.F., 2008, p. 8).

Respecto a la autorregulación emocional, podemos definirla como un sistema de control que supervisa que nuestra experiencia emocional se ajusta a nuestras metas de referencia y forma parte de la inteligencia emocional e influyen en aspectos como la empatía, el control de impulsos, la resolución de problemas, el control de la ira, el reconocimiento de similitudes y diferencias entre las personas, los procesos de comunicación y relación interpersonales, o los procesos de afrontamiento de situaciones estresantes (Vived Conte, E., 2011, p.p. 46 y 47).

En torno a la inteligencia emocional, Goleman (2012) señala como sus características:

a) Capacidad de motivarnos a nosotros mismos.

b) Perseverar en el empeño a pesar de las posibles frustraciones.

c) Controlar los impulsos.

d) Diferir las gratificaciones. 
e) Regular nuestros propios estados de ánimo.

f) Evitar que la angustia interfiera con nuestras facultades racionales.

g) Capacidad de empatizar y confiar en los demás (p.p. 25 y 26).

John Mayer y Peter Salovey señalaron que a través de la habilidad emocional se puede:

a) Percibir y expresar con exactitud las emociones.

b) Tener acceso a las emociones ajenas o generales cuando éstas sean productivas para el pensamiento.

c) Entender la naturaleza de las emociones, de manera que estemos en capacidad de regularlas con el fin de promover el crecimiento tanto emocional como intelectual (Como se citó en López de Bernal, M.E. y González Medina, M.F., 2008, p. 8).

Conforme a Kautz, las habilidades sociales y emocionales como capacidades individuales pueden:

a) Manifestarse en patrones congruentes de pensamientos, sentimientos y comportamientos.

b) Desarrollarse mediante experiencias de aprendizaje formales e informales.

c) Ser factores impulsores importantes de los resultados socioeconómicos a lo largo de la vida de la persona" (Como se citó en OCDE, 2016, p. 35).

\section{CURRÍCULO}

Para Mallarino Flórez, C. (2007), el currículo, situado contextualmente, se puede entender como el ejercicio cotidiano de construcción de sentidos, fundamentos, principios y criterios de desempeño, el lugar de reflexión, evaluación y confrontación de imaginarios y realidades, la instancia de apropiación de es- trategias metodológicas y comunicativas, y la dinámica dialógica en el proceso de estructuración y fortalecimiento de concepciones y paradigmas (p. 75). En ese entendido, el currículo es la guía de la labor docente.

Y en opinión de Suárez Díaz, Reinaldo (2005), el currículo es el conjunto de oportunidades, experiencias de aprendizaje planeadas o no, explícitas o implícitas, que el estudiante encuentra en la escuela para su propio desarrollo intelectual, personal y social. De igual manera, el autor de referencia, distingue entre currículo explícito o manifiesto y currículo implícito u oculto. El primero, está constituido por las normas, políticas, contenidos mínimos obligatorios, planes de estudio, programas académicos y requisitos exigidos por una entidad educativa en vista de la aprobación de un curso o la opción de un título. El segundo, se refiere a todas aquellas experiencias consciente $o$ inconscientemente vividas, valores reforzados, actitudes y destrezas adquiridas mediante las interacciones que se suceden cotidianamente en la entidad educativa de manera espontánea, no programadas en forma oficial e intencional, si bien inconscientemente pueden descubrirse en la elección y orientación implícita de los planes de estudio (p.p. 159 y 160).

En tanto, Gimeno Sacristán \& Pérez, A. (1996) plantean la perspectiva del currículo como configurador de la práctica educativa y conceptúan que desde este enfoque el currículo se centra en la dialéctica Teoría-Práctica como el esquema integrador de los problemas de la práctica educativa escolarizada.

Y la concepción de un currículo como configurador de una práctica social escolar, obliga a escrutar las condiciones en que se produce y debe contribuir a una mejora de la comprensión de los fenómenos educativos y a comprometerse con la intervención de la realidad para transformarla (Osorio Villegas, M., 2017, p. 150). 
En este entendido, si se favorece el desarrollo de las habilidades socioemocionales a través del currículo de la educación superior, se puede lograr mejorar los entornos educativos, y por réplica, el social.

\section{EDUCACIÓN SUPERIOR}

Ibáñez, B. C. (1994) considera que "la educación es un proceso de conocimiento enseñado de manera seriada, por lo cual se ha dado lugar a su clasificación en niveles, como el preescolar, básico, medio, medio superior y el superior" (p. 99).

Conforme al tercer párrafo del artículo 37 de la LGE, la educación superior es la que se imparte después del bachillerato o de sus equivalentes. Y está compuesta por la licenciatura, la especialidad, la maestría y el doctorado, así como por opciones terminales previas a la conclusión de la licenciatura. Además, comprende la educación normal en todos sus niveles y especialidades.

Para Guerrero Barrios, J. y Faro Resendiz, M.T. (2012:39), la función de las Instituciones de Educación Superior (IES) está dirigida a propiciar un ambiente educativo que además de solucionar problemas sociales actuales junto con los alumnos, también ayude a preparar mejores personas para el futuro.

En razón de lo anterior, se piensa que la educación superior es un excelente área de oportunidad para formar individuos que mejoren los contextos sociales a través de una convivencia armónica.

\section{CONVIVENCIA}

Según el Diccionario de la Lengua Española (2018), la palabra "convivencia" hace alusión a la "acción de convivir". Y este término proviene del latín conviverre, que significa "vivir en compañía de otro u otros".
En ese entendido, convivir significa: "vivir unos con otros basándonos en una determinadas relaciones sociales y en unos códigos valorativos en el marco de un contexto social" (Jares, Xesús, R., 2006:17). Por su parte, la convivencia se refiere "a la manera como vivimos colectivamente". (Arango Cálad, Carlos, A., 2001:88).

\section{DESARROLLO DE HABILIDADES SOCIOEMOCIONALES EN ALUMNOS DE EDUCACIÓN SUPERIOR COMO CONTRIBUCIÓN PARA UNA MEJOR CONVIVENCIA}

En México, tanto la CPEUM como la LGE fundamentan la contribución de la educación para una mejor convivencia humana y para el desarrollo del individuo en los siguientes términos:

El inciso c de la fracción II del artículo $3^{\circ}$ de la Constitución Política de México, determina que: "el criterio que orientará a esa educación contribuirá a la mejor convivencia humana".

Por su parte, el párrafo segundo del artículo $2^{\circ}$ de la la LGE señala: "La educación es medio fundamental para adquirir, transmitir y acrecentar la cultura; es proceso permanente que contribuye al desarrollo del individuo y a la transformación de la sociedad...".

La fracción I del artículo $7^{\circ}$ de la ley en cita indica que la educación que impartan el Estado, sus organismos descentralizados y los particulares con autorización o con reconocimiento de validez oficial de estudios tendrá como fin: "Contribuir al desarrollo integral del individuo, para que ejerza plena y responsablemente sus capacidades humanas".

Por su parte, la fracción III del artículo $8^{\circ}$ de la ley en cometo prescribe que el criterio que orientará a la educación "contribuirá a la mejor convivencia humana". 
En ese entendido, se considera que el desarrollo de habilidades socioemocionales, forma parte del desarrollo integral del individuo. Y la educación superior, podría contribuir a una mejor convivencia de las personas, desarrollando en sus alumnos dichas habilidades.

Así, podemos afirmar que el desarrollo de habilidades socioemocionales en alumnos de educación superior puede contribuir a una mejor convivencia.

La afirmación anterior se soporta en el Informe: "Habilidades para el progreso social" (2016), de la Organización para la Cooperación y el Desarrollo Económicos (OCDE), el cual a través de una investigación analítica, da cuenta sobre el poder de las habilidades socioemocionales. En él se incluyen revisiones de la literatura, análisis empíricos de datos longitudinales y una revisión de las políticas y prácticas de los países miembros de la OCDE y las economías asociadas.

En el Informe de referencia, la OCDE afirma que las habilidades socioemocionales tienen efectos de envergadura sobre diversos aspectos sociales. $\mathrm{Y}$ a este respecto concluye que éstas pueden contribuir a:

- Reducir el comportamiento antisocial.

- Proteger a los individuos contra la posibilidad de ser victimizados por comportamientos agresivos.

- Reducir la probabilidad de que los individuos se involucren en problemas de conducta.

- Reducir no sólo la probabilidad de que las personas cometan agresiones, sino también permitirles evitar ser las víctimas de éstas.

- Mejorar la vida de la gente al optimizar sus comportamientos y estilos de vida.

Los resultados aquí vertidos, advierten de los beneficios que se obtienen al promover el desarrollo de habilidades socioemocionales en las personas, porque éstas nos permiten relacionarnos con las personas de una manera más sana y por ende, con ello se propicia una convivencia más armónica.

La relevancia de las habilidades socioemocionesenlas relacionesinterpersonales es doble. Aportan una relación valiosa para dar significado a la relación e influencian la propia interacción (MEPSD, 2008:18).

El desarrollo de habilidades socioemocionales en alumnos de educación superior puede contribuir a una mejor convivencia, en virtud de que se si se mejoran las relaciones sociales en ese entorno, se pueden replicar en el ámbito social.

Para la OCDE (2016), la educación puede contribuir a aportar una diversidad de habilidades que empoderan a los individuos para enfrentar mejor los desafíos de la vida diaria. Habilidades sociales y emocionales como la perseverancia, la estabilidad emocional y la sociabilidad también son importantes para lograr resultados positivos. Estas habilidades permiten a las personas traducir intenciones en actos, establecer relaciones positivas con la familia, los amigos y la comunidad y evitar caer en estilos de vida poco saludables y comportamientos riesgosos (p. 23)

\section{VÍAS PARA FOMENTAR LAS HABILIDADES SOCIOEMOCIONALES EN EL CURRÍCULO ESCOLAR DE LA EDUCACIÓN SUPERIOR}

La propuesta de esbozo de las rutas para fomentar las habilidades socioemocionales en el currículum escolar de la educación superior debe seguir los mismos ejes que lo conforman.

En este sentido, Suárez Díaz, Reinaldo (2005: p. 160), señala que el currículum escolar está conformado por: 
- Las políticas institucionales expresadas.

- El ambiente físico, psicológico, social o mundo de vida de la institución educativa.

- Planes de estudio, estrategias, métodos y técnicas utilizadas para el logro de sus objetivos.

- Los programas específicos de cada una de las disciplinas.

De ahí que se plantean un esbozo de acciones educativas en los siguientes ejes:

\section{POLÍTICAS INSTITUCIONALES}

- Diseñar y aplicar evaluaciones diagnósticas para detectar necesidades relacionadas con desarrollo de habilidades socioemocionales.

- Incluir en los planes de desarrollo institucionales IES, propuestas de intervención para el impulso de habilidades socioemocionales en los programas y proyectos educativos que lo conforman.

- Diseñar manuales de convivencia y llevar a cabo una amplia difusión de éstos entre la comunidad universitaria.

\section{AMBIENTES DE LA INSTITUCIÓN EDUCATIVA}

- Fomentar entre los colaboradores de las IES, el buen manejo de las habilidades socioemocionales como parte de un adecuado clima organizacional, para lo cual se puede impartir formación y capacitación al respecto.

\section{PLAN DE ESTUDIOS}

- Incluir temas para favorecer el desarrollo de habilidades socioemocionales en la mayoría de los programas de asignaturas.

- Incluir estrategias, métodos y técnicas para el logro de sus objetivos con enfoque de desarrollo de habilidades socioemocionales.

\section{PROGRAMAS DE LAS ASIGNATURAS}

- Diseñar programas de asignatura con temáticas específicas en materia de habilidades socioemocionales.

\section{DISCUSIÓN}

El fomento de habilidades socioemocionales contribuye en el desarrollo integral del individuo. $Y$ en la educación superior se pueden desarrollar o fortalecer tales habilidades para incidir en mejores formas de relación y convivencia.

Las IES en México pueden tener un papel preponderante en la transformación de una mejor sociedad y robustecer su currículo escolar con herramientas que nos permitan transitar de situaciones desfavorecidas a un futuro más esperanzador.

México ha vivido en los últimos años convulsiones sin precedente que han convertido su unidad y su cohesión en preocupaciones colectivas de primer orden. Cuando la violencia deviene en problema social, como nos ha ocurrido a nosotros, hay que volver la vista hacia la escuela como espacio privilegiado para la convivencia (Landeros, Leticia y Chávez Concepción, 2015: 13).

La educación siempre será la panacea para mejorar, entre otras cosas, nuestra forma de relacionarnos con los otros. La propia LGE en la fracción III de su artículo $8^{\circ}$ describe que el criterio que orientará a la educación contribuirá a la mejor convivencia humana, tanto por los elemetos que aportorte para:

"robustecer en el educando, junto con el aprecio para la dignidad de la persona y la integridad de la familia, la convicción del interés general de la sociedad, cuanto por el cuidado que ponga en sustentar los ideales de fraternidad e igualdad de dere- 
chos de todos los hombres, evitando los privilegios de razas, de religión, de grupos, de sexos o de individuos".

Así, el desarrollo de habilidades socioemocionales en alumnos de educación superior puede contribuir a una mejor convivencia, porque permiten fortalecer nuestros vínculos afectivos y sociales con los otros, y con ello, se puede lograr una mejor convivencia entre las personas.

\section{CONCLUSIONES}

La legislación mexicana fundamenta la contribución de la educación para una mejor convivencia humana y para el desarrollo del individuo.

- El desarrollo de habilidades socioemocionales forma parte del desarrollo integral del individuo. Y la educación superior, podría contribuir a una mejor convivencia de las personas, desarrollando en sus alumnos dichas habilidades socioemocionales.

- El desarrollo de habilidades socioemocionales en alumnos de educación superior puede contribuir a una mejor convivencia, toda vez que se si se mejoran las relaciones sociales en ese entorno, se pueden replicar en el ámbito social.

A través del currículo escolar de la educación superior se puede fomentar el desarrollo de habilidades socioemocionales.

\section{REFERENCIAS BIBLIOGRÁFICAS}

Adam, E. et. al. (2007). Emociones y educación. ¿Qué son y cómo intervenir desde la escuela? $2^{\mathrm{a}}$ edición, Barcelona: GRAÓ.

Arango Cálad, Carlos, A. (2001). Hacia una psicología de la convivencia, Revista Colombiana de Psicología, (10), 79-89.

BID. (2017). Aprender mejor. Políticas públicas para el desarrollo de habilidades, Washington D.C: BID.

De la Mora Ledesma, J. G. (1990). Psicología del Aprendizaje. Formas 2. México: Progreso.

García Rojas, A. Daniel. (2010). Estudio sobre la asertividad y las habilidades sociales en el alumnado de Educación Social. Revista de Educación (12), Universidad de Huelva, 225240.

Gimeno Sacristán \& Pérez, A. (1996). Comprender y transformar la enseñanza. Madrid: Morata.

Goleman, D. (2010). Inteligencia emocional. España: Kairos S.A.

Guerrero Barrios, J. y Faro Resendiz, M.T. (2012), Breve análisis del concepto de Educación Superior, Revista Alternativas en Psicología, (27), 34-41.

Ibáñez, B. C. (1994). Pedagogía y Psicología Interconductual. Revista Mexicana de Análisis de la Conducta, (20), 99-112.

Jares, Xesús, R. (2006). Pedagogía de la convivencia, Barcelona: Graó.

Landeros, Leticia y Chávez Concepción (2015). Convivencia y disciplina en la escuela, México, INEE.

López de Bernal, M.E. y González Medina, M.F. (2008). Inteligencia emocional, Colombia: GAMMA, S.A. 
Mallarino Flórez, C. (2007). La contextualización del currículo: Cognición y no verbalidad, Revista Científica Guillermo de Ockham, Vol. 5, No. 1, Enero-Junio.

Ministerio de Educación, Cultura y Deporte (MECD) (1998). Habilidades Sociales en el Currículo, España: Centro de Investigación y Documentación Educativa. España: MECD.

Monereo, C. (Coord.). (1998). Estrategias de Enseñanza y Aprendizaje- Formación de Enseñanza y Aprendizaje. Formación del Profesorado y Aplicación en el Aula. México-España: Grao.

OCDE. (2016). Habilidades para el progreso social. El poder de las habilidades sociales y emocionales, Montreal: UNESCO-UIS.

Osorio Villegas, M. (2017). El currículo: Perspectivas para acercarnos a su comprensión, Revista Zona Próxima, Núm. 26, enero-junio, pp. 140-151.

Ribes Iñesta, E. (2002). Psicología del Aprendizaje. México: Manual Moderno.

Segura Morales, M. y Arcas Cuenca, M. (2007). Educar las emociones y los sentimientos. Introducción práctica al complejo mundo de las emociones, Madrid: Narcea.

Suárez Díaz, R. (2009). La Educación. Estrategias de Enseñanza Aprendizaje. Teorías Educativas. México: Trillas.

Trianes Torres, M. V., y Gallardo Cruz, J. A. (Coords.) (2008). Psicología de la educación y del desarrollo en contexto escolares. Madrid: Pirámide.

Vived Conte, E. (2011). Habilidades sociales, autonomía personal y autorregulación, Zaragoza: Prensas Universitarias de Zaragoza.
Zavala Berbena, et. al. (2008). Inteligencia emocional y habilidades sociales en adolescentes con alta aceptación social, Revista Electrónica de Investigación Psicoeducativa. No 15, Vol 6 (2), 319- 338.

\section{Legislación}

Constitución Política de los Estados Unidos Mexicanos.

Ley General del Educación.

Páginas electrónicas

http://www.rae.es 